\title{
Spontaneous CP Violating Phase as The CKM Matrix Phase
}

\author{
Shao-Long Chen ${ }^{1}$, N. G. Deshpande ${ }^{2}$, Xiao-Gang He ${ }^{1}$, Jing Jiang ${ }^{2}$ and Lu-Hsing Tsai ${ }^{1}$ \\ ${ }^{1}$ Department of Physics and Center for Theoretical Sciences, \\ National Taiwan University, Taipei, Taiwan \\ ${ }^{2}$ Institute of Theoretical Science, University of Oregon, Eugene, OR 97403, USA
}

(Dated: October 24, 2018)

\begin{abstract}
We propose that the $\mathrm{CP}$ violating phase in the CKM mixing matrix is identical to the $\mathrm{CP}$ phases responsible for the spontaneous CP violation in the Higgs potential. A specific multi-Higgs model with Peccei-Quinn (PQ) symmetry is constructed to realize this idea. The CP violating phase does not vanish when all Higgs masses become large. There are flavor changing neutral current (FCNC) interactions mediated by neutral Higgs bosons at the tree level. However, unlike general multi-Higgs models, the FCNC Yukawa couplings are fixed in terms of the quark masses and CKM mixing angles. Implications for meson-anti-meson mixing, including recent data on $D-\bar{D}$ mixing, and neutron electric dipole moment (EDM) are studied. We find that the neutral Higgs boson masses can be at the order of one hundred GeV. The neutron EDM can be close to the present experimental upper bound.
\end{abstract}




\section{INTRODUCTION}

The origin of $\mathrm{CP}$ violation is one of the outstanding problems of modern particle physics. There have been several experimental measurements of CP violation 1]. All of them are consistent with the Cabbibo-Kobayashi-Maskawa (CKM) model [2, 3], where the source

of CP violation comes from the phase [3] $\delta_{K M}$ in the CKM mixing matrix for quarks. A successful model of CP violation at the leading order should have the successful features of the CKM model. It is important to understand the origin of $\mathrm{CP}$ violation. An interesting proposal due to T.-D. Lee was that CP is spontaneously violated [4]. The popular Weinberg model [5] of spontaneous $\mathrm{CP}$ violation model has problems [6, 7] with data and has been decisively ruled out by CP violating measurement in B decays [1]. Spontaneous CP violation in the Left-Right models has also been ruled out for the same reason[8]. In this work we restore the idea that $\mathrm{CP}$ is broken spontaneously and the phase $\delta_{K M}$ is the same as the phase $\delta_{\text {spon }}$ that causes spontaneous $\mathrm{CP}$ violation in the Higgs potential. We construct specific models to realize this idea. The main difference of our models lies in how the CP violating phase in the CKM matrix is identified [9].

Let us start by describing how a connection between $\delta_{K M}$ and $\delta_{\text {spon }}$ can be made. It is well known that to have spontaneous CP violation, one needs two or more Higgs doublets $\phi_{i}$. Consider the following Yukawa couplings with multi-Higgs doublets,

$$
L_{Y}=\bar{Q}_{L}\left(\Gamma_{u 1} \phi_{1}+\Gamma_{u 2} \phi_{2}\right) U_{R}+\bar{Q}_{L} \Gamma_{d} \tilde{\phi}_{d} D_{R}+\text { h.c. }
$$

where $Q_{L}, U_{R}$ and $D_{R}$ are the left-handed doublet, right-handed up and right-handed down quarks, respectively. Generation indices are suppressed. $\tilde{\phi}_{d}=-i \sigma_{2} \phi_{d}^{*}$ and $\phi_{d}$ may be one of the $\phi_{1,2}$ or another doublet Higgs field. The Yukawa couplings $\Gamma_{u 1, u 2, d}$ must be real if CP is only violated spontaneously.

The Higgs doublets when expressed in terms of the component fields and their vacuum expectation values (vev) $v_{i}$ are given by

$$
\phi_{i}=e^{i \theta_{i}} H_{i}=e^{i \theta_{i}}\left(\begin{array}{c}
\frac{1}{\sqrt{2}}\left(v_{i}+R_{i}+i A_{i}\right) \\
h_{i}^{-}
\end{array}\right) .
$$

The quark mass terms in the Lagrangian are

$$
L_{m}=-\bar{U}_{L}\left[M_{u 1} e^{i \theta_{1}}+M_{u 2} e^{i \theta_{2}}\right] U_{R}-\bar{D}_{L} M_{d} e^{-i \theta_{d}} D_{R}+\text { h.c. }
$$


where $M_{u i}=-\Gamma_{u i} v_{i} / \sqrt{2}$.

The phases $\theta_{1}$ and $\theta_{d}$ can be absorbed by redefining the fields $U_{R}$ and $D_{R}$. However, the phase difference $\delta=\theta_{2}-\theta_{1}$ cannot be removed and it depends on the Higgs potential. A non-zero $\delta$ indicates spontaneous $\mathrm{CP}$ violation, $\delta=\delta_{\text {spon }}$. Without loss of generality, we work in the basis where $D_{L}, D_{R}$ are already in their mass eigenstates. In this basis the down quark mass matrix $M_{d}$ is diagonalized, which will be indicated by $\hat{M}_{d}$. In general the up quark mass matrix $M_{u}=M_{u 1}+e^{i \delta} M_{u 2}$ is not diagonal. Diagonalizing $M_{u}$ produces the CKM mixing matrix. One can write $\hat{M}_{u}=V_{C K M} M_{u} V_{R}^{\dagger}$. Here $V_{C K M}$ is the CKM matrix and $V_{R}$ is an unknown unitary matrix. A direct identification of the phase $\delta_{\text {spon }}$ with the phase $\delta_{K M}$ in the CKM matrix is not possible in general at this level. There are, however, classes of mass matrices which allow such a connection. A simple example is provided by setting $V_{R}$ to be the unit matrix. With this condition, $M_{u}=V_{C K M}^{\dagger} \hat{M}_{u}$. One then needs to show that $V_{C K M}^{\dagger}$ can be written as

$$
V_{C K M}^{\dagger}=\left(M_{u 1}+e^{i \delta} M_{u 2}\right) \hat{M}_{u}^{-1}
$$

Expressing the CKM matrix in this form is very suggestive. If $V_{C K M}$ (or $V_{C K M}^{\dagger}$ ) can always be written as a sum of two terms with a relative phase, then the phase in the CKM matrix can be identified with the phase $\delta$.

We now demonstrate that it is the case by using the Particle Data Group (PDG) parametrization as an example. To get as close as to the form in Eq. (4), we write the PDG CKM matrix as [1]

$$
V_{C K M}=\left(\begin{array}{lll}
e^{-i \delta_{13}} & 0 & 0 \\
0 & 1 & 0 \\
0 & 0 & 1
\end{array}\right)\left(\begin{array}{lll}
c_{12} c_{13} e^{i \delta_{13}} & s_{12} c_{13} e^{i \delta_{13}} & s_{13} \\
-s_{12} c_{23}-c_{12} s_{23} s_{13} e^{i \delta_{13}} & c_{12} c_{23}-s_{12} s_{23} s_{13} e^{i \delta_{13}} & s_{23} c_{13} \\
s_{12} s_{23}-c_{12} c_{23} s_{13} e^{i \delta_{13}} & -c_{12} s_{23}-s_{12} c_{23} s_{13} e^{i \delta_{13}} & c_{23} c_{13}
\end{array}\right),
$$

where $s_{i j}=\sin \theta_{i j}$ and $c_{i j}=\cos \theta_{i j}$.

Absorbing the left matrix into the definition of $U_{L}$ field, we have

$$
M_{u 1}=\left(\begin{array}{ccc}
0 & -s_{12} c_{23} & s_{12} s_{23} \\
0 & c_{12} c_{23} & -c_{12} s_{23} \\
s_{13} & s_{23} c_{13} & c_{23} c_{13}
\end{array}\right) \hat{M}_{u}, \quad M_{u 2}=\left(\begin{array}{ccc}
c_{12} c_{13} & -c_{12} s_{23} s_{13} & -c_{12} c_{23} s_{13} \\
s_{12} c_{13} & -s_{12} s_{23} s_{13} & -s_{12} c_{23} s_{13} \\
0 & 0 & 0
\end{array}\right) \hat{M}_{u},
$$

and $\delta=-\delta_{13}$. We therefore find that it is possible to identify the CKM phase with that resulting from spontaneous $\mathrm{CP}$ violation. Note that as long as the phase $\delta$ is not zero, CP 
violation will show up in the charged currents mediated by $\mathrm{W}$ exchange. The effects do not disappear even when Higgs boson masses are all set to be much higher than the $W$ scale. Furthermore, $M_{1,2}$ are fixed in terms of the CKM matrix elements and the quark masses, as opposed to being arbitrary in general multi-Higgs models.

We comment that the solution is not unique even when $V_{R}$ is set to be the unit matrix. To see this, one can take another parametrization for the CKM matrix, such as the original Kobayashi-Maskawa (KM) matrix [3]. More physical requirements are needed to uniquely determine the connection. The phenomenological consequences will therefore be different. We will come back to this when we look at phenomenology of models. The key point we want to establish is that there are solutions where the phase in the CKM matrix can be identified with the phase causing spontaneous CP violation in the Higgs potential.

The mass matrices $M_{u 1}$ and $M_{u 2}$ can be written in a parametrization independent way in terms of the eigen-mass matrix $\hat{M}_{u}$, the CKM matrix, and the phase $\delta$,

$$
\begin{aligned}
& M_{u 1}=V_{C K M}^{\dagger} \hat{M}_{u}-\frac{e^{i \delta}}{\sin \delta} \operatorname{Im}\left(V_{C K M}^{\dagger}\right) \hat{M}_{u}, \\
& M_{u 2}=\frac{1}{\sin \delta} \operatorname{Im}\left(V_{C K M}^{\dagger}\right) \hat{M}_{u} .
\end{aligned}
$$

Alternatively, a model can be constructed with two Higgs doublets couple to the down sector and one Higgs doublet couples to the up sector to have,

$$
L_{Y}=\bar{Q}_{L} \Gamma_{u} \phi_{u} U_{R}+\bar{Q}_{L}\left(\Gamma_{d 1} \tilde{\phi}_{1}+\Gamma_{d 2} \tilde{\phi}_{2}\right) D_{R}+\text { h.c. } .
$$

In this case $M_{d i}=-\Gamma_{d i} v_{i} / \sqrt{2}$, and

$$
\begin{aligned}
& M_{d 1}=V_{C K M} \hat{M}_{d}+\frac{e^{-i \delta}}{\sin \delta} \operatorname{Im}\left(V_{C K M}\right) \hat{M}_{d}, \\
& M_{d 2}=-\frac{1}{\sin \delta} \operatorname{Im}\left(V_{C K M}\right) \hat{M}_{d} .
\end{aligned}
$$

We denote the above two possibilities as Model a) with two Higgs doublets coupled to the up sector, and Model b) with two Higgs doublets coupled to the down sector.

\section{MODEL BUILDING}

A common problem for models with spontaneous $\mathrm{CP}$ violation is that a strong QCD $\theta$ term will be generated [7]. Constraint from neutron dipole moment measurement will rule 
out spontaneous CP violation as the sole source if there is no mechanism to make sure that the $\theta$ term is small enough if not zero. The models mentioned above face the same problem. We therefore supplement the model with a Peccei-Quinn (PQ) symmetry [10] to ensure a small $\theta$.

To have spontaneous CP violation and also PQ symmetry simultaneously, more than two Higgs doublets are needed [11]. For our purpose we find that in order to have spontaneous $\mathrm{CP}$ violation with PQ symmetry at least three Higgs doublets $\phi_{i}=e^{i \theta_{i}} H_{i}$ and one complex Higgs singlet $\tilde{S}=e^{i \theta_{s}} S=e^{i \theta_{s}}\left(v_{s}+R_{s}+i A_{s}\right) / \sqrt{2}$ are required. The Higgs singlet with a large vacuum expectation value (vev) renders the axion from PQ symmetry breaking to be invisible [12, 13], thus satisfying experimental constraints on axion couplings to fermions. We will henceforth work with models with an invisible axion [12].

The PQ charges for Models a) and b) are as follows

$$
\begin{aligned}
& \text { Model a) } \quad Q_{L}: 0, \quad U_{R}:-1, D_{R}:-1, \phi_{1,2}:+1, \phi_{d}=\phi_{3}:-1 \text {; } \\
& \text { Model b) } Q_{L}: 0, U_{R}:+1, D_{R}:+1, \phi_{1,2}:+1, \phi_{u}=\phi_{3}:-1 \text {. }
\end{aligned}
$$

In both cases, $\tilde{S}$ has PQ charge +2 . For leptons, the PQ charges can have different assignments. For example: $L_{L}: 0, e_{R}:-1$ or $L_{L}: 0, e_{R}:+1$.

For both models a) and b), the Higgs potentials have the same form which is given by

$$
\begin{aligned}
V & =-m_{1}^{2} H_{1}^{\dagger} H_{1}-m_{2}^{2} H_{2}^{\dagger} H_{2}-m_{3}^{2} H_{3}^{\dagger} H_{3}-m_{12}^{2}\left(H_{1}^{\dagger} H_{2} e^{i\left(\theta_{2}-\theta_{1}\right)}+\text { h.c. }\right)-m_{s}^{2} S^{\dagger} S \\
& +\lambda_{1}\left(H_{1}^{\dagger} H_{1}\right)^{2}+\lambda_{2}\left(H_{2}^{\dagger} H_{2}\right)^{2}+\lambda_{t}\left(H_{3}^{\dagger} H_{3}\right)^{2}+\lambda_{s}\left(S^{\dagger} S\right)^{2} \\
& +\lambda_{3}\left(H_{1}^{\dagger} H_{1}\right)\left(H_{2}^{\dagger} H_{2}\right)+\lambda_{3}^{\prime}\left(H_{1}^{\dagger} H_{1}\right)\left(H_{3}^{\dagger} H_{3}\right)+\lambda_{3}^{\prime \prime}\left(H_{2}^{\dagger} H_{2}\right)\left(H_{3}^{\dagger} H_{3}\right) \\
& +\lambda_{4}\left(H_{1}^{\dagger} H_{2}\right)\left(H_{2}^{\dagger} H_{1}\right)+\lambda_{4}^{\prime}\left(H_{1}^{\dagger} H_{3}\right)\left(H_{3}^{\dagger} H_{1}\right)+\lambda_{4}^{\prime \prime}\left(H_{2}^{\dagger} H_{3}\right)\left(H_{3}^{\dagger} H_{2}\right) \\
& +\frac{1}{2} \lambda_{5}\left(\left(H_{1}^{\dagger} H_{2}\right)^{2} e^{i 2\left(\theta_{2}-\theta_{1}\right)}+\text { h.c. }\right)+\lambda_{6}\left(H_{1}^{\dagger} H_{1}\right)\left(H_{1}^{\dagger} H_{2} e^{i\left(\theta_{2}-\theta_{1}\right)}+\text { h.c. }\right) \\
& +\lambda_{7}\left(H_{2}^{\dagger} H_{2}\right)\left(H_{1}^{\dagger} H_{2} e^{i\left(\theta_{2}-\theta_{1}\right)}+h . c .\right)+\lambda_{8}\left(H_{3}^{\dagger} H_{3}\right)\left(H_{1}^{\dagger} H_{2} e^{i\left(\theta_{2}-\theta_{1}\right)}+h . c .\right) \\
& +f_{1} H_{1}^{\dagger} H_{1} S^{\dagger} S+f_{2} H_{2}^{\dagger} H_{2} S^{\dagger} S+f_{3} H_{3}^{\dagger} H_{3} S^{\dagger} S+d_{12}\left(H_{1}^{\dagger} H_{2} e^{i\left(\theta_{2}-\theta_{1}\right)}+H_{2}^{\dagger} H_{1} e^{-i\left(\theta_{2}-\theta_{1}\right)}\right) S^{\dagger} S \\
& +f_{13}\left(H_{1}^{\dagger} H_{3} S e^{i\left(\theta_{3}+\theta_{s}-\theta_{1}\right)}+\text { h.c. }\right)+f_{23}\left(H_{2}^{\dagger} H_{3} S e^{i\left(\theta_{3}+\theta_{s}-\theta_{2}\right)}+\text { h.c. }\right) .
\end{aligned}
$$

Only two phases occur in the above expression, which we choose to be $\delta=\theta_{2}-\theta_{1}$ and $\delta_{s}=\theta_{3}+\theta_{s}-\theta_{2}$. The phase $\theta_{3}+\theta_{s}-\theta_{1}$ can be written as $\delta+\delta_{s}$. Differentiating with respect to $\delta_{s}$ to get one of the conditions for minimization of the potential, we get

$$
f_{13} v_{1} v_{3} v_{s} \sin \left(\delta_{s}+\delta\right)+f_{23} v_{2} v_{3} v_{s} \sin \delta_{s}=0
$$


We see that $\delta$ and $\delta_{s}$ are related with

$$
\tan \delta_{s}=-\frac{f_{13} v_{1} \sin \delta}{f_{23} v_{2}+f_{13} v_{1} \cos \delta} .
$$

Therefore, $\delta$ is the only independent phase in the Higgs potential. A non-zero $\sin \delta$ is the source of spontaneous $\mathrm{CP}$ violation and also the only source of $\mathrm{CP}$ violation in the model.

In this model the Goldstone fields $h_{w}$ and $h_{z}$ that are "eaten" by $W$ and $Z$, and the axion field are given by

$$
\begin{aligned}
& h_{w}=\frac{1}{v}\left(v_{1} h_{1}^{-}+v_{2} h_{2}^{-}+v_{3} h_{3}^{-}\right), \\
& h_{z}=\frac{1}{v}\left(v_{1} A_{1}+v_{2} A_{2}+v_{3} A_{3}\right), \\
& a=\left(-v_{1} v_{3}^{2} A_{1}-v_{2} v_{3}^{2} A_{2}+v_{12}^{2} v_{3} A_{3}-v^{2} v_{s} A_{s}\right) / N_{a},
\end{aligned}
$$

where $v^{2}=v_{1}^{2}+v_{2}^{2}+v_{3}^{2}$ and $N_{a}^{2}=\left(v_{12}^{2} v_{3}^{2} v^{2}+v^{4} v_{s}^{2}\right)$ with $v_{12}^{2}=v_{1}^{2}+v_{2}^{2}$.

We remove $h_{w}$ and $h_{z}$ in the Yukawa interaction by making the following changes of basis,

$$
\begin{aligned}
\left(\begin{array}{c}
A_{1} \\
A_{2} \\
A_{3} \\
A_{s}
\end{array}\right)=\left(\begin{array}{cccc}
v_{2} / v_{12} & -v_{1} v_{3} v_{s} / N_{A} & v_{1} / v & -v_{1} v_{3}^{2} / N_{a} \\
-v_{1} / v_{12} & -v_{2} v_{3} v_{s} / N_{A} & v_{2} / v & -v_{2} v_{3}^{2} / N_{a} \\
0 & v_{12}^{2} v_{s} / N_{A} & v_{3} / v & v_{12}^{2} v_{3} / N_{a} \\
0 & v_{12}^{2} v_{3} / N_{A} & 0 & -v^{2} v_{s} / N_{a}
\end{array}\right)\left(\begin{array}{c}
a_{1} \\
a_{2} \\
h_{z} \\
a
\end{array}\right), \\
\left(\begin{array}{c}
h_{1}^{-} \\
h_{2}^{-} \\
h_{3}^{-}
\end{array}\right)=\left(\begin{array}{ccc}
v_{2} / v_{12} & v_{1} v_{3} / v v_{12} & v_{1} / v \\
-v_{1} / v_{12} & v_{2} v_{3} / v v_{12} & v_{2} / v \\
0 & -v_{12} / v & v_{3} / v
\end{array}\right)\left(\begin{array}{c}
H_{1}^{-} \\
H_{2}^{-} \\
h_{w}
\end{array}\right)
\end{aligned}
$$

where $N_{A}^{2}=v_{12}^{2}\left(v_{12}^{2} v_{3}^{2}+v_{s}^{2} v^{2}\right) . \quad a_{1,2}$ and $H_{1,2}^{-}$are the physical degrees of freedom for the Higgs fields. With the same rotation as that for the neutral pseudoscalar, the neutral scalar Higgs fields $\left(R_{1}, R_{2}, R_{3}, R_{s}\right)^{T}$ become $\left(H_{1}^{0}, H_{2}^{0}, H_{3}^{0}, H_{4}^{0}\right)^{T}$. Since the invisible axion scale $v_{s}$ is much larger than the electroweak scale, to a very good approximation, $N_{a}=v^{2} v_{s}$ and $N_{A}=v_{12} v v_{s}$.

In the rotated basis described above, we have the Yukawa interactions for physical Higgs degrees of freedom as the following

$$
\begin{aligned}
L_{Y}^{(a)} & =\bar{U}_{L}\left[\hat{M}_{u} \frac{v_{1}}{v_{12} v_{2}}-\left(\hat{M}_{u}-V_{C K M} \operatorname{Im}\left(V_{C K M}^{\dagger}\right) \hat{M}_{u} \frac{e^{i \delta}}{\sin \delta}\right) \frac{v_{12}}{v_{1} v_{2}}\right] U_{R}\left(H_{1}^{0}+i a_{1}^{0}\right) \\
& +\bar{U}_{L} \hat{M}_{u} U_{R}\left[\frac{v_{3}}{v_{12} v}\left(H_{2}^{0}+i a_{2}\right)-\frac{1}{v} H_{3}^{0}+\frac{v_{3}^{2}}{v^{2} v_{s}}\left(H_{4}^{0}+i a\right)\right]
\end{aligned}
$$




$$
\begin{aligned}
& -\bar{D}_{L} \hat{M}_{d} D_{R}\left[\frac{v_{12}}{v_{3} v}\left(H_{2}^{0}-i a_{2}\right)+\frac{1}{v} H_{3}^{0}+\frac{v_{12}^{2}}{v^{2} v_{s}}\left(H_{4}^{0}-i a\right)\right] \\
& +\sqrt{2} \bar{D}_{L}\left[V_{C K M}^{\dagger} \hat{M}_{u} \frac{v_{1}}{v_{2} v_{12}}-\left(V_{C K M}^{\dagger} \hat{M}_{u}-\operatorname{Im}\left(V_{C K M}^{\dagger}\right) \hat{M}_{u} \frac{e^{i \delta}}{\sin \delta}\right) \frac{v_{12}}{v_{1} v_{2}}\right] U_{R} H_{1}^{-} \\
& -\sqrt{2} \frac{v_{3}}{v_{12} v} \bar{D}_{L} V_{C K M}^{\dagger} \hat{M}_{u} U_{R} H_{2}^{-}-\sqrt{2} \frac{v_{12}}{v v_{3}} \bar{U}_{L} V_{C K M} \hat{M}_{d} D_{R} H_{2}^{+}+h . c . \\
L_{Y}^{(b)} & =\bar{D}_{L}\left[\hat{M}_{d} \frac{v_{1}}{v_{12} v_{2}}-\left(\hat{M}_{d}+V_{C K M}^{\dagger} \operatorname{Im}\left(V_{C K M}\right) \hat{M}_{d} \frac{e^{-i \delta}}{\sin \delta}\right) \frac{v_{12}}{v_{1} v_{2}}\right] D_{R}\left(H_{1}^{0}-i a_{1}^{0}\right) \\
& +\bar{D}_{L} \hat{M}_{d} D_{R}\left[\frac{v_{3}}{v_{12} v}\left(H_{2}^{0}-i a_{2}\right)-\frac{1}{v} H_{3}^{0}+\frac{v_{3}^{2}}{v^{2} v_{s}}\left(H_{4}^{0}-i a\right)\right] \\
& -\bar{U}_{L} \hat{M}_{u} U_{R}\left[\frac{v_{12}}{v_{3} v}\left(H_{2}^{0}+i a_{2}\right)+\frac{1}{v} H_{3}^{0}+\frac{v_{12}^{2}}{v^{2} v_{s}}\left(H_{4}^{0}+i a\right)\right] \\
& -\sqrt{2} \bar{U}_{L}\left[V_{C K M} \hat{M}_{d} \frac{v_{1}}{v_{2} v_{12}}-\left(V_{C K M} \hat{M}_{d}+I_{m}\left(V_{C K M}\right) \hat{M}_{d} \frac{e^{-i \delta}}{\sin \delta}\right) \frac{v_{12}}{v_{1} v_{2}}\right] D_{R} H_{1}^{+} \\
& +\sqrt{2} \frac{v_{3}}{v_{12} v} \bar{U}_{L} V_{C K M} \hat{M}_{d} D_{R} H_{2}^{+}+\sqrt{2} \frac{v_{12}}{v v_{3}} \bar{D}_{L} V_{C K M}^{\dagger} \hat{M}_{u} U_{R} H_{2}^{-}+h . c .
\end{aligned}
$$

Note that the couplings of $a$ and $H_{4}^{0}$ to quarks are suppressed by $1 / v_{s}$, and that only the exchange of $H_{1}^{0}$ and $a_{1}^{0}$ can induce tree level FCNC interactions. The FCNC couplings are proportional to $V_{C K M} \operatorname{Im}\left(V_{C K M}^{\dagger}\right) \hat{M}_{u}$ and $V_{C K M}^{\dagger} \operatorname{Im}\left(V_{C K M}\right) \hat{M}_{d}$ for models a) and b), respectively.

We have mentioned before that the identification of the phase $\delta$ with that in the CKM matrix does not uniquely determine the full Yukawa coupling. Here we give two often used parameterizations, the PDG CKM matrix and the original KM matrix with the CP violating phase indicated by $\delta_{K M}$, to illustrate the details. In the two cases under consideration, the phase $\delta$ are identified with $-\delta_{13}$ and $-\delta_{K M}$, respectively. The differences will show up in the FCNC of neutral Higgs coupling to quarks which are proportional to the following quantities,

PDG : $\quad V_{C K M} \operatorname{Im}\left(V_{C K M}^{\dagger}\right) \hat{M}_{u}=-\sin \delta_{13} e^{i \delta_{13}}\left(\begin{array}{ccc}c_{13}^{2} & -s_{23} s_{13} c_{13} & -c_{23} s_{13} c_{13} \\ -s_{23} s_{13} c_{13} & s_{23}^{2} s_{13}^{2} & s_{23} c_{23} s_{13}^{2} \\ -c_{23} s_{13} c_{13} & s_{23} c_{23} s_{13}^{2} & c_{23}^{2} s_{13}^{2}\end{array}\right) \hat{M}_{u}$, $V_{C K M}^{\dagger} \operatorname{Im}\left(V_{C K M}\right) \hat{M}_{d}=\sin \delta_{13} e^{-i \delta_{13}}\left(\begin{array}{ccc}c_{12}^{2} & s_{12} c_{12} & 0 \\ s_{12} c_{12} & s_{12}^{2} & 0 \\ 0 & 0 & 0\end{array}\right) \hat{M}_{d}$

$\mathrm{KM}: \quad V_{C K M} \operatorname{Im}\left(V_{C K M}^{\dagger}\right) \hat{M}_{u}=-\sin \delta_{K M} e^{i \delta_{K M}}\left(\begin{array}{ccc}0 & 0 & 0 \\ 0 & s_{2}^{2} & -s_{2} c_{2} \\ 0 & -s_{2} c_{2} & c_{2}^{2}\end{array}\right) \hat{M}_{u}$ 


$$
V_{C K M}^{\dagger} \operatorname{Im}\left(V_{C K M}\right) \hat{M}_{d}=\sin \delta_{K M} e^{-i \delta_{K M}}\left(\begin{array}{ccc}
0 & 0 & 0 \\
0 & s_{3}^{2} & -s_{3} c_{3} \\
0 & -s_{3} c_{3} & c_{3}^{2}
\end{array}\right) \hat{M}_{d}
$$

\section{MESON AND ANTI-MESON MIXING AND NEUTRON EDM}

In this section we study some implications for meson and anti-meson mixing and neutron electric dipole moment.

\section{A. Meson and Anti-meson Mixing}

Meson and anti-meson mixing has been observed previously in $K^{0}-\bar{K}^{0}, B_{d, s}^{0}-\bar{B}_{d, s}^{0}$ [1] and in $D^{0}-\bar{D}^{0}$ very recently [14]. In the models considered in the previous section, besides the standard "box" diagram contributions to the mixing due to $W$ exchange, there are also tree level contributions due to the FCNC interactions of $H_{1}^{0}$ and $a_{1}$.

The interaction Lagrangian for $H_{l}$ and $a_{k}$ with quarks have the following form for both models a) and b),

$$
L=\bar{q}_{i}\left(a_{i j}^{l}+b_{i j}^{l} \gamma_{5}\right) q_{j} H_{l}^{0}+i \bar{q}_{i}\left(c_{i j}^{k}+d_{i j}^{k} \gamma_{5}\right) q_{j} a_{k}
$$

For the meson and anti-meson mixing, only the FCNC interaction terms of $H_{1}^{0}$ and $a_{1}$ contribute. We can write $a^{1}=d^{1}=\alpha$ and $b^{1}=c^{1}=\beta$, with $\alpha=\left(A+A^{\dagger}\right) / 2$ and $\beta=\left(A-A^{\dagger}\right) / 2$, and $A$ given by:

$$
\begin{aligned}
& \text { For a }), \quad A=V_{C K M} \operatorname{Im}\left(V_{C K M}^{\dagger}\right) \hat{M}_{u} \frac{e^{i \delta}}{\sin \delta} \frac{v_{12}}{v_{1} v_{2}} ; \\
& \text { For b), } A=-V_{C K M}^{\dagger} \operatorname{Im}\left(V_{C K M}\right) \hat{M}_{d} \frac{e^{-i \delta}}{\sin \delta} \frac{v_{12}}{v_{1} v_{2}} .
\end{aligned}
$$

Using the definition $<0 \mid \bar{q}_{i} \gamma^{\mu} \gamma_{5} q_{j}>=i f_{P} p_{P}^{\mu} / \sqrt{2 m_{P}}$ and the equation of motion $\bar{q}_{i} \gamma_{5} q_{j}=$ $\left(p_{i}-p_{j}\right)^{\mu} \bar{q}_{i} \gamma_{\mu} \gamma_{5} q_{j} /\left(m_{i}+m_{j}\right)$ with $p^{P}=p_{j}-p_{i}$, we obtain the matrix element for $P-\bar{P}$ mixing in the vacuum saturation approximation as

$$
\begin{aligned}
M_{12}= & \frac{1}{m_{H_{1}}^{2}}\left[\left(b_{i j}^{2}-\frac{1}{12}\left(a_{i j}^{2}+b_{i j}^{2}\right)\right) \frac{f_{P}^{2} m_{P}^{3}}{\left(m_{i}+m_{j}\right)^{2}}+\frac{1}{12}\left(b_{i j}^{2}-a_{i j}^{2}\right) f_{p}^{2} m_{P}\right] \\
& -\frac{1}{m_{a_{1}}^{2}}\left[\left(a_{i j}^{2}-\frac{1}{12}\left(a_{i j}^{2}+b_{i j}^{2}\right)\right) \frac{f_{P}^{2} m_{P}^{3}}{\left(m_{i}+m_{j}\right)^{2}}+\frac{1}{12}\left(a_{i j}^{2}-b_{i j}^{2}\right) f_{P}^{2} m_{P}\right] \\
& +\frac{i 2 m_{H_{1} a_{1}}^{2}}{m_{H_{1}}^{2} m_{a_{1}}^{2}} \frac{5 a_{i j} b_{i j}}{6} \frac{f_{P}^{2} m_{P}^{3}}{\left(m_{i}+m_{j}\right)^{2}} .
\end{aligned}
$$


where $m_{H_{1} a_{1}}^{2}$ parameterizes the mixing between $a_{1}$ and $H_{1}$, that is determined from the Higgs potential $V=m_{H_{1} a_{1}}^{2} H_{1} a_{1}+\ldots$. Since $m_{H_{1} a_{1}}^{2}$ involves new parameters, it can be made small enough to avoid any conflict with data. We will neglect their contributions when discussing meson and anti-meson mixing. We will come back to this when discussing neutron EDM.

It is obvious from the structure of the flavor changing coupling in Eq. (17) that the flavor changing structure for the PDG and KM cases are different. For PDG case, in model a) there is tree level contribution from neutral Higgs exchange to $D^{0}-\bar{D}^{0}$ mixing, but no contribution to $K^{0}, B_{d}^{0}$ and $B_{s}^{0}$ mixing. In model b), there is only non-zero contribution to $K^{0}-\bar{K}^{0}$ mixing at the tree level. For the KM case, there is no tree level contribution to meson mixing in model a). For model b), there is only non-zero contribution to $B_{s}^{0}$ mixing.

In our numerical analysis, we will use the following values for the relevant parameters. For the CKM matrix elements, we take the PDG central values with [1]: $s_{12}=0.227$, $s_{23}=0.042, s_{13}=0.004$ and $\sin \delta_{13}=0.84$ (equivalently $s_{1}=0.227, s_{2}=0.0358$, $s_{3}=0.0176$ and $\sin \delta=0.97$ for the KM parameterization). For the quark masses, we take [16] $m_{u}(1 \mathrm{GeV})=5 \mathrm{MeV}, m_{d}(1 \mathrm{GeV})=10 \mathrm{MeV}, m_{s}(1 \mathrm{GeV})=187 \mathrm{MeV}, m_{c}\left(m_{c}\right)=$ $1.30 \mathrm{GeV}, m_{b}\left(m_{b}\right)=4.34 \mathrm{GeV}, m_{t}=174 \mathrm{GeV}$. For the meson decay constants, we take [17] $f_{K}=156 \mathrm{MeV}, f_{D}=201 \mathrm{MeV}$, and $f_{B_{s}}=260 \mathrm{MeV}$.

\section{$\underline{\text { Models with PDG parameterization }}$}

We consider the models with PDG parameterization first.

Model a): In this case there is mixing only in $D^{0}-\bar{D}^{0}$ system. Combining the BaBar and Belle [14] results the $68 \%$ C.L. range for $x=\Delta m / \Gamma_{D}$ is determined to be $(5.5 \pm 2.2) \times 10^{-3}$ [15]. Theoretically the elements in $A$ for this particular case are $A_{12}=-s_{23} s_{13} c_{13} \frac{m_{c} v_{12}}{v_{1} v_{2}}$ and $A_{21}=-s_{23} s_{13} c_{13} \frac{m_{u} v_{12}}{v_{1} v_{2}}$, which implies that $a_{12} \sim b_{12} \sim-s_{23} s_{13} c_{13} \frac{m_{c} v_{12}}{2 v_{1} v_{2}}$. We obtain

$$
\begin{aligned}
x & \approx \frac{5}{12} s_{23}^{2} s_{13}^{2} c_{13}^{2}\left(\frac{v_{12} m_{c}}{v_{1} v_{2}}\right)^{2} \frac{f_{D}^{2} m_{D}}{\Gamma_{D}}\left(\frac{m_{D}}{m_{c}+m_{u}}\right)^{2}\left(\frac{1}{m_{H_{1}}^{2}}-\frac{1}{m_{a_{1}}^{2}}\right) \\
& =7.5 \times 10^{-5} \frac{1}{(\sin 2 \beta)^{2} v_{12}^{2}}\left(\frac{1}{m_{H_{1}}^{2}}-\frac{1}{m_{a_{1}}^{2}}\right)(100 \mathrm{GeV})^{4} .
\end{aligned}
$$

where $\tan \beta$ is defined to be $v_{1} / v_{2}$.

It is well known that the SM short distance contribution to the $D-\bar{D}$ mixing is small. Long distance contributions can be much larger, but they suffer from considerable uncertainty. New physics may contribute significantly [15]. It is tempting to see if the 
new contribution in this model can account for the full measured value. If the effective neutral Higgs mass $m_{\text {eff }}^{2}=1 /\left(1 / m_{H_{1}}^{2}-1 / m_{a_{1}}^{2}\right)$ is of order $100 \mathrm{GeV}$, one would require $\sin ^{2} 2 \beta v_{12}^{2} \sim(12)^{2} \mathrm{GeV}^{2}$. Since $v_{1,2}$ are related to the top quark mass, with the assumption that the top quark Yukawa coupling $y_{t} \leq 1$, one of them should be large, $\sim 240 \mathrm{GeV}$. Saturating the experimental central value for $x$, we would have $\sin (2 \beta) \sim 0.05$ implying $v_{1} / v_{2}$ or $v_{2} / v_{1}$ to be of the order of $1 / 40$. If all vevs are the same order of magnitude, the new contribution does not produce large enough $x$ to saturate the measured value.

Model b): In this case there is mixing only in $K^{0}-\bar{K}^{0}$ system. We have

$$
\frac{\Delta m_{K}}{m_{K}}=4.4 \times 10^{-12} \frac{1}{\sin ^{2} 2 \beta v_{12}^{2}}\left(\frac{1}{m_{H_{1}}^{2}}-\frac{1}{m_{a_{1}}^{2}}\right)(100 \mathrm{GeV})^{4}
$$

This is to be compared with the experimental data $\Delta m_{K} / m_{K}=7.0 \times 10^{-15}$. It puts strong constraints on the scalar masses. i.e., the Higgs particles must be at least $\mathrm{TeV}$ scale to suppress the value if $a_{1}$ and $H_{1}$ are not degenerate in mass.

\section{Models with KM parameterization}

We now come to models with the original KM parameterization. In this case, there is no meson and anti-meson mixing in Model a).

Model b): There is mixing only in $B_{s}-\bar{B}_{s}$ system. We have

$$
\frac{\Delta m_{B_{S}}}{m_{B_{s}}}=9.5 \times 10^{-12} \frac{1}{\sin ^{2} 2 \beta v_{12}^{2}}\left(\frac{1}{m_{H_{1}}^{2}}-\frac{1}{m_{a_{1}}^{2}}\right)(100 \mathrm{GeV})^{4}
$$

Experimental value $\Delta m_{B_{s}}=17.4 \mathrm{ps}^{-1}$ implies $\Delta m_{B_{s}} / m_{B_{s}}=2.1 \times 10^{-12}$. It has been shown in Ref. [18] that the New Physics contribution to $\Delta m_{B_{s}}$ can be up to $10 \%$. To obtain the lowest Higgs boson mass, we maximize $\sin 2 \beta=1$ which requires $v_{1}=v_{2}$. Taking $v_{1,2,3}$ to be all equal, the Higgs boson mass can be as low as $300 \mathrm{GeV}$. With smaller $v_{1,2}$ or non-equal $v_{1}$ and $v_{2}$ would lead to more stringent bound on Higgs mass.

\section{B. The neutron EDM}

The neutron EDM can also provide much information on the model parameters. The standard model predicts a very small [19] $d_{n}\left(<10^{-31} e \mathrm{~cm}\right)$. The present experimental upper bound on neutron EDM $d_{n}$ is very tight [1]: $\left|d_{n}\right|<0.63 \times 10^{-25} e \mathrm{~cm}$. We now 
study whether neutron EDM can reach its present bound after imposing the constraints from meson and anti-meson mixing discussed in the previous section.

In the models we are studying, the quark EDMs will be generated at loop levels due to mixing between $a_{i}$ and $H_{i}$. From Higgs potential given earlier, we find the mixing parameters,

$$
\begin{aligned}
m_{H_{1} a_{1}}^{2}= & {\left[\left(\lambda_{6}-\lambda_{7}\right) v_{1} v_{2}-\lambda_{5}\left(v_{1}^{2}-v_{2}^{2}\right) \cos \delta\right] \sin \delta } \\
m_{H_{1} a_{2}}^{2} \simeq & -\frac{f_{13} \sin \left(\delta+\delta_{s}\right) v v_{s}}{\sqrt{2} v_{2}}, \\
m_{H_{2} a_{1}}^{2} \simeq & \frac{1}{2 v_{2} v}\left[-2 \lambda_{5} v_{1} v_{3} v_{2}^{2} \sin 2 \delta+2\left(-\lambda_{6} v_{1}^{2}-\lambda_{7} v_{2}^{2}+\left(\lambda_{8}+d_{12}\right) v_{12}^{2}\right) v_{2} v_{3} \sin \delta\right. \\
& \left.+\sqrt{2} f_{13} v^{2} v_{s} \sin \left(\delta+\delta_{s}\right)\right], \\
m_{H_{3} a_{1}}^{2}= & \frac{v_{12}}{v}\left[2 \lambda_{5} v_{1} v_{2} \cos \left(\theta_{1}-\theta_{2}\right)+\lambda_{6} v_{1}^{2}+\lambda_{7} v_{2}^{2}+\lambda_{8} v_{3}^{2}\right] \sin \delta .
\end{aligned}
$$

Note that all the parameters above are zero if $\sin \delta=0$.

The one loop contributions to the neutron EDM are suppressed for the usual reason of being proportional to light quarks masses to the third power for diagram in which the internal quark is the same as the external quark. In model a) with PDG parameterization, there is a potentially large contribution when there is a top quark in the loop. However, the couplings to top are proportional to $s_{13}$, therefore the contribution to neutron EDM is much smaller than the present upper bound. We will not discuss them further.

It is well known that exchange of Higgs at the two loop level may be more important than the one loop contribution, through the quark EDM $O_{q}^{\gamma}[20]$, quark color EDM $O_{q}^{C}$ [20], and the gluon color EDM $O_{g}^{C}[21]$ defined as

$$
O_{q}^{\gamma}=-\frac{d_{q}}{2} i \bar{q} \sigma_{\mu \nu} \gamma_{5} F^{\mu \nu} q, \quad O_{q}^{C}=-\frac{f_{q}}{2} i g_{s} \bar{q} \sigma_{\mu \nu} \gamma_{5} G^{\mu \nu} q, \quad O_{g}^{C}=-\frac{1}{6} C f_{a b c} G_{\mu \nu}^{a} G_{\mu \alpha}^{b} \tilde{G}_{\nu \alpha}^{c},
$$

where $F^{\mu \nu}$ is the photon field strength, $G^{\mu \nu}$ is the gluon field strength and $\tilde{G}^{\mu \nu}=\frac{1}{2} \epsilon_{\mu \nu \alpha \beta} G^{\alpha \beta}$.

In the valence quark model, the quark EDM and color EDM contributions to the neutron EDM $d_{n}$ are given by [19]

$$
d_{n}^{\gamma}=\eta_{d}\left[\frac{4}{3} d_{d}-\frac{1}{3} d_{u}\right]_{\Lambda}, \quad d_{n}^{C}=e \eta_{f}\left[\frac{4}{9} f_{d}+\frac{2}{9} f_{u}\right]_{\Lambda}
$$

where $[22] \eta_{d}=\left(\frac{\alpha_{s}\left(M_{Z}\right)}{\alpha_{s}\left(m_{b}\right)}\right)^{16 / 23}\left(\frac{\alpha_{s}\left(m_{b}\right)}{\alpha_{s}\left(m_{c}\right)}\right)^{16 / 25}\left(\frac{\alpha_{s}\left(m_{c}\right)}{\alpha_{s}(\Lambda)}\right)^{16 / 27} \approx 0.166$ and $\eta_{f}=$ $\left(\frac{\alpha_{s}\left(M_{Z}\right)}{\alpha_{s}\left(m_{b}\right)}\right)^{14 / 23}\left(\frac{\alpha_{s}\left(m_{b}\right)}{\alpha_{s}\left(m_{c}\right)}\right)^{14 / 25}\left(\frac{\alpha_{s}\left(m_{c}\right)}{\alpha_{s}(\Lambda)}\right)^{14 / 27} \frac{\alpha_{s}\left(M_{Z}\right)}{\alpha_{s}(\Lambda)} \approx 0.0117$ are the QCD running factors from scale $m_{Z}$ to the hadron scale $\Lambda$. 
A naive dimensional analysis (NDA) estimate gives the gluon color EDM contribution to the neutron EDM as the following

$$
d_{n} \approx \frac{e M}{4 \pi} \xi C,
$$

where $M=4 \pi f_{\pi}=1190 \mathrm{MeV}$ is the scale of chiral symmetry breaking. The QCD running factor is [23] $\xi=\left(\frac{g(\Lambda)}{4 \pi}\right)^{3}\left(\frac{\alpha_{s}\left(m_{b}\right)}{\alpha_{s}\left(m_{t}\right)}\right)^{-54 / 23}\left(\frac{\alpha_{s}\left(m_{c}\right)}{\alpha_{s}\left(m_{b}\right)}\right)^{-54 / 25}\left(\frac{\alpha_{s}(\Lambda)}{\alpha_{s}\left(m_{c}\right)}\right)^{-54 / 27} \approx 1.2 \times 10^{-4}$.

The two loop contribution to $d_{q}, f_{q}$ and $C$ are given by

$$
d_{q}=\frac{e \alpha_{e m} Q_{q}}{24 \pi^{3}} m_{q} G(q), \quad f_{q}=\frac{\alpha_{s}}{64 \pi^{3}} m_{q} G(q), \quad C=\frac{1}{8 \pi} H(g),
$$

where $Q_{q}$ is the charge of $q$ quark and

$$
\begin{aligned}
G(q) & =\left[\left(f\left(\frac{m_{t}^{2}}{m_{H_{l}}^{2}}\right)-f\left(\frac{m_{t}^{2}}{m_{a_{k}}^{2}}\right)\right) \operatorname{Im} Z_{t q}^{l k}+\left(g\left(\frac{m_{t}^{2}}{m_{H_{l}}^{2}}\right)-g\left(\frac{m_{t}^{2}}{m_{a_{k}}^{2}}\right)\right) I m Z_{q t}^{l k}\right], \\
H(g) & =\left(h\left(\frac{m_{t}^{2}}{m_{H_{l}}^{2}}\right)-h\left(\frac{m_{t}^{2}}{m_{a_{k}}^{2}}\right)\right) I m Z_{t t}^{l k},
\end{aligned}
$$

where $\operatorname{Im} Z_{i j}$ is defined through $\operatorname{Im} Z_{i j}^{l k}=2 a_{i i}^{l} d_{j j}^{k} \lambda_{l k} /\left(m_{i} m_{j}\right)$ with $a^{l}, d^{k}$ defined by Eq. (18) and $\lambda_{l k}=m_{H_{l} a_{k}}^{2} /\left(m_{H_{l}}^{2}-m_{a_{k}}^{2}\right)$ is a mixing factor depending on the neutral Higgs bosons exchanged in the loop.

The functions $f(z), g(z)$ and $h(z)$ are given by

$$
\begin{aligned}
f(z) & =\frac{z}{2} \int_{0}^{1} d x \frac{1-2 x(1-x)}{x(1-x)-z} \ln \frac{x(1-x)}{z}, \\
g(z) & =\frac{z}{2} \int_{0}^{1} d x \frac{1}{x(1-x)-z} \ln \frac{x(1-x)}{z}, \\
h(z) & =\frac{z^{2}}{2} \int_{0}^{1} d x \int_{0}^{1} d u \frac{u^{3} x^{3}(1-x)}{[z x(1-u x)+(1-u)(1-x)]^{2}} .
\end{aligned}
$$

Numerically we find that functions $(f, g, h)$ change slowly from $(0.5,1,0.1)$ to $(0.2,0.2,0.03)$ when Higgs masses are increased from $100 \mathrm{GeV}$ to $1 \mathrm{TeV}$.

\section{Models with PDG parameterization}

Model a): The 2-loop contributions to the neutron EDM due to the Higgs bosons exchange in the loop are proportional to the mixing factor $\lambda_{l k}(f, g, h)$. We take these factors to be approximately equal to estimate the contributions from different Higgs exchange.

If using the parameters which produce $D$ mixing, i.e., $\tan \beta=40, v_{12} \sim 240 \mathrm{GeV}$ and $v_{3} \sim 10 \mathrm{GeV}$ and Higgs around $100 \mathrm{GeV}$ are used, we find that the dominant contribution 
is from $H_{3}, a_{1}$ exchange,

$$
d_{n} \approx-1.5 \times 10^{-25} \frac{m_{H_{3} a_{1}}^{2}}{m_{H_{3}}^{2}-m_{a_{1}}^{2}} \mathrm{e} \mathrm{cm} .
$$

If all vevs are of the same order, i.e., taking $v_{1}=v_{2}=v_{3}$ with Higgs mass of order 100 $\mathrm{GeV}$, we have

$$
d_{n} \approx 8 \times 10^{-26} \frac{m_{H_{3} a_{1}}^{2}}{m_{H_{3}}^{2}-m_{a_{1}}^{2}} \mathrm{e} \mathrm{cm} .
$$

Model b): In this case $H_{1}, a_{1}$ do not couple to $\bar{t} t$, so the two loop contribution to quark EDM and quark and gluon color EDM from the $H_{1}, a_{1}$ are small.

The contributions to neutron EDM are about the same from the $H_{1}, a_{2}$ and $H_{2,3}, a_{1}$ exchange, with different mixing factors. Explicitly as an example, for the case $H_{1}, a_{2}$ exchange with the Higgs mass taken to be $1 \mathrm{TeV}$, as high as allowed by $K^{0}-\bar{K}^{0}$ mixing, we have

$$
d_{n} \approx-1 \times 10^{-26} \frac{m_{H_{1} a_{2}}^{2}}{m_{H_{1}}^{2}-m_{a_{2}}^{2}} \mathrm{e} \mathrm{cm} .
$$

If $m_{H_{1} a_{2}}^{2}$ is not too much smaller than $m_{H_{1}, a_{2}}^{2}$, the neutron EDM can be close to the upper bound.

$\underline{\text { Models with KM parameterization }}$

Model a): In this case there are no constraints from meson mixing, the Higgs mass can be low. If all vevs are of the same order, i.e. taking $v_{1}=v_{2}=v_{3}$ with Higgs mass of order $100 \mathrm{GeV}$, we have the main contribution come from $H_{1}, a_{2}$ exchange,

$$
d_{n} \approx 5 \times 10^{-26} \frac{m_{H_{1} a_{2}}^{2}}{m_{H_{1}}^{2}-m_{a_{2}}^{2}} \mathrm{e} \mathrm{cm} .
$$

Model b): Similar to the case for Model b) as in the PDG parameterization case, the contributions from the $H_{1}, a_{1}$ exchange are small. Taking the vevs to be same order and Higgs mass to be of the order of $100 \mathrm{GeV}$, we find the contributions from $H_{1}, a_{2}$ exchange and $H_{2,3}, a_{1}$ exchange are comparable. For the case $H_{1}, a_{2}$ exchange, the contribution is given by

$$
d_{n} \approx 5 \times 10^{-26} \frac{m_{H_{1} a_{2}}^{2}}{m_{H_{1}}^{2}-m_{a_{2}}^{2}} \mathrm{e} \mathrm{cm} .
$$

If one takes the Higgs mass to be $300 \mathrm{GeV}$ as that from $B_{s}-\bar{B}_{s}$ mixing, the neutron EDM will be smaller. 


\section{DISCUSSIONS AND CONCLUSIONS}

In our previous discussions, we have not considered Yukawa coupling for the lepton sector. An analogous study can be carried out. If one introduces right handed neutrinos, see-saw mechanism can be applied to generate small neutrino masses if the axion scale $v_{s}$ is identified with the see-saw scale. We briefly discuss two classes of models parallel to the quark sector before conclusion.

Model a): The PQ charges for lepton doublet $L_{L}$, electron $e_{R}$ and neutrino $\nu_{R}$ are assigned as: $L_{L}(0), e_{R}(-1)$ and $\nu_{R}(-1)$. The Yukawa couplings are then

$$
L=\bar{L}_{L}\left(Y_{1} H_{1}+Y_{2} H_{2} e^{i \delta}\right) \nu_{R}+\bar{L}_{L} Y_{3} \tilde{H}_{3} e_{R}+\bar{\nu}_{R}^{C} Y_{s} S e^{i\left(\delta+\delta_{s}\right)} \nu_{R}+\text { h.c. }
$$

In this case the mass matrices in $L_{m}=-\bar{e}_{L} M_{e} e_{R}-\bar{\nu}_{L} M_{D} \nu_{R}-(1 / 2) \bar{\nu}_{R}^{C} M_{R} \nu_{R}$ can be written as

$$
M_{l}=-\frac{1}{\sqrt{2}} Y_{3} v_{3}, \quad M_{D}=-\frac{1}{\sqrt{2}}\left(Y_{1} v_{1}+Y_{2} v_{2} e^{i \delta}\right), \quad M_{R}=-\sqrt{2} Y_{s} v_{s} e^{i\left(\delta+\delta_{s}\right)} .
$$

The charged current mixing matrix in the lepton sector, the Pontecove-Maki-NakagawaSakata (PMNS) matrix [24], $V_{P M N S}$ similar to the $V_{C K M}$ matrix is given by $V_{P M N S}=V_{L}^{e} V_{L}^{\nu \dagger}$, where $V_{L}^{e}$ and $V_{L}^{\nu}$ are defined by: $M_{e}=V_{L}^{e \dagger} \hat{M}_{e} V_{R}^{e}$ and $M_{\nu}=-M_{D} M_{R}^{-1} M_{D}^{T}=V_{L}^{\nu \dagger} \hat{M}_{\nu} V_{L}^{\nu *}$ with $\hat{M}_{e}$ and $\hat{M}_{\nu}$ the charge lepton and light neutrino eigen-mass matrices.

Model b): The PQ charges for lepton doublet $L_{L}$, electron $e_{R}$ and neutrino $\nu_{R}$ are assigned as: $L_{L}(0), e_{R}(+1)$ and $\nu_{R}(+1)$. The Yukawa couplings are

$$
L=\bar{L}_{L} Y_{3} H_{3} \nu_{R}+\bar{L}_{L}\left(Y_{1} \tilde{H}_{1}+Y_{2} \tilde{H}_{2} e^{-i \delta}\right) e_{R}+\bar{\nu}_{R}^{C} Y_{s} S^{\dagger} e^{-i\left(\delta+\delta_{s}\right)} \nu_{R}+\text { h.c. }
$$

and

$$
M_{l}=-\frac{1}{\sqrt{2}}\left(Y_{1} v_{1}+Y_{2} v_{2} e^{-i \delta}\right), \quad M_{D}=-\frac{1}{\sqrt{2}} Y_{3} v_{3}, \quad M_{R}=-\sqrt{2} Y_{s} v_{s} e^{-i\left(\delta+\delta_{s}\right)} .
$$

From the above we see that, in general there are $\mathrm{CP}$ violation in the mixing matrix $V_{P M N S}$, and the source is the same as that in the Higgs potential. But the identification of the phase $\delta$ with the phase in the $V_{P M N S}$ becomes more complicated due to the appearance of $M_{R}$. The related details will be discussed elsewhere.

We have proposed that the $\mathrm{CP}$ violating phase in the CKM mixing matrix to be the same as that causing spontaneous CP violation in the Higgs potential. Specific multiHiggs doublet models have been constructed to realize this idea. There are flavor changing 
neutral current mediated by neutral Higgs bosons at the tree level. However, even when the Higgs boson masses are set to be very large, the phase in the CKM matrix can be made finite and $\mathrm{CP}$ violating effects will not disappear unlike in other models of spontaneous $\mathrm{CP}$ violation where the $\mathrm{CP}$ violation disappear when Higgs boson masses become large. Another interesting feature of this model is that the FCNC Yukawa couplings are fixed in terms of the quark masses and CKM mixing angles, making phenomenological analysis much easier.

We have studied some implications for meson-anti-meson mixing, including recent data on $D-\bar{D}$ mixing, and neutron electric dipole moment. We find that the neutral Higgs boson masses can be at the order of $100 \mathrm{GeV}$. The neutron EDM can be close to the present experimental upper bound.

Acknowledgments This work was supported in part by the National Science Council and the National Center for Theoretical Sciences, and by the U.S. Department of Energy under Grants No DE-FG02-96ER40969. We thank Jon Parry for pointing out a typo in our first version on the arXiv.

[1] W-M Yao et al 2006 J. Phys. G: Nucl. Part. Phys. 331.

[2] N. Cabbibo, Phys. Rev. Lett. 10, 531(1963).

[3] M. Kobayashi and T. Maskawa, Prog. Theor. Phys. 49, 652 (1973).

[4] T. D. Lee, Phys. Rev. D 8, 1226 (1973); T. D. Lee, Phys. Rept. 9, 143 (1974).

[5] S. Weinberg, Phys. Rev. Lett. 37, 657 (1976); G. C. Branco, Phys. Rev. Lett. 44, 504 (1980).

[6] D. Chang, X. G. He and B. H. J. McKellar, Phys. Rev. D 63, 096005 (2001) arXiv:hep-ph/9909357]; G. Beall and N. G. Deshpande, Phys. Lett. B 132, 427 (1983); I. I. Y. Bigi and A. I. Sanda, Phys. Rev. Lett. 58, 1604 (1987).

[7] R. Akhoury and I. I. Y. Bigi, Nucl. Phys. B 234, 459 (1984).

[8] P. Ball, J. M. Frere and J. Matias, Nucl. Phys. B 572, 3 (2000) arXiv:hep-ph/9910211.

[9] G. C. Branco and R. N. Mohapatra, Phys. Lett. B 643, 115 (2006) arXiv:hep-ph/0607271]; G. C. Branco, D. Emmanuel-Costa and J. C. Romao, Phys. Lett. B 639, 661 (2006) arXiv:hep-ph/0604110.

[10] R. D. Peccei and H. R. Quinn, Phys. Rev. D 16, 1791 (1977): R. D. Peccei and H. R. Quinn, Phys. Rev. Lett. 38, 1440 (1977). 
[11] X. G. He and R. R. Volkas, Phys. Lett. B 208, 261 (1988) [Erratum-ibid. B 218, 508 (1989)]; C. Q. Geng, X. D. Jiang and J. N. Ng, Phys. Rev. D 38, 1628 (1988).

[12] A.R. Zhitnitsky, Sov. J. Nucl. Phys. 31, 260(1980); M. Dine, W. Fischler and M. Srednicki, Phys. Lett. B 104, 199 (1981).

[13] J. E. Kim, Phys. Rev. Lett. 43 (1979) 103; M. Shifman, A. Vainshtein, V. Zakharov, Nucl. Phys. B166 (1980) 493.

[14] B. Aubert et al. [BaBar Collaboration], arXiv: hep-ex/0703020, BABAR-PUB-07/019, SLACPUB-12385, SCIPP-07/01; M. Staric, Talk presented at XLII Rencontres de Moriond, La Thuile, Italy, 10-17 March, 2007; K. Abe et al. [Belle Collaboration], arXiv: hep-ex/0703036.

[15] M. Chiuchini, et al arXiv:hep-ph/0703204; X. G. He and G. Valencia, arXiv:hep-ph/0703270; C. H. Chen, C. Q. Geng and T. C. Yuan, arXiv:0704.0601 [hep-ph]; Z. z. Xing and S. Zhou, arXiv:0704.0971 [hep-ph]; P. Ball, arXiv:0704.0786 [hep-ph]; K. Babu et al., Phys. Lett. B 205, 540 (1988).

[16] H. Fusaoka and Y. Koide, Phys. Rev. D 57, 3986 (1998) arXiv:hep-ph/9712201.

[17] M. Okamoto, PoS(LAT2005)013 arXiv:hep-lat/0510113.

[18] A. Lenz and U. Nierste, arXiv: hep-ph/0612167; X. G. He and G. Valencia, Phys. Rev. D 74, 013011 (2006) arXiv:hep-ph/0605202; K. Cheung, C. W. Chiang, N. G. Deshpande and J. Jiang, arXiv:hep-ph/0604223.

[19] N. G. Deshpande, G. Eilam and W. L. Spence, Phys. Lett. B 108, 42 (1982); X. G. He, B. H. J. McKellar and S. Pakvasa, Int. J. Mod. Phys. A 4, 5011 (1989) [Erratum-ibid. A 6, 1063 (1991)]; B. H. J. McKellar, S. R. Choudhury, X. G. He and S. Pakvasa, Phys. Lett. B 197, 556 (1987).

[20] S. M. Barr and A. Zee, Phys. Rev. Lett. 65, 21 (1990) [Erratum-ibid. 65, 2920 (1990)]; J.F. Gunion and D. Wyler, Phys. Lett. B 248, 170 (1990).

[21] S. Weinberg, Phys. Rev. Lett. 63, 2333 (1989); Phys. Rev. D42, 860 (1990).

[22] D. Chang, W.-Y. Keung, and T. C. Yuan, Phys. Lett. B 251, 608 (1990); D. Chang, et al., Phys. Rev. D 46, 3876 (1992).

[23] E. Braaten, C.-S. Li, and T.-C. Yuan, Phys. Rev. Lett. 64, 1709 (1990); Phys. Rev. D 42, 276 (1990).

[24] B. Pontecovo, Sov. Phys. JETP 6, 429(1957); Z. Maki, M. Nakagawa and S. Sakata, Prog. Theor. 28, 870(1962). 